\title{
Braiding of the Attractor and the Failure of Iterative Algorithms
}

\section{Citation}

McMullen, Curtis T. 1988. Braiding of the attractor and the failure of iterative algorithms. Inventiones Mathematicae 91(2): 259-272.

\section{Published Version}

doi:10.1007/BF01389368

\section{Permanent link}

http://nrs.harvard.edu/urn-3:HUL.InstRepos:3597231

\section{Terms of Use}

This article was downloaded from Harvard University's DASH repository, and is made available under the terms and conditions applicable to Other Posted Material, as set forth at http:// nrs.harvard.edu/urn-3:HUL.InstRepos:dash.current.terms-of-use\#LAA

\section{Share Your Story}

The Harvard community has made this article openly available.

Please share how this access benefits you. Submit a story.

Accessibility 


\section{Braiding of the attractor and the failure of iterative algorithms *}

\section{Curt McMullen}

Department of Mathematics, Princeton University, Princeton, NJ 08540, USA

\section{Introduction}

The motion of the attractor in a stable family of rational maps determines a group of braids on the Riemann sphere. We show the corresponding group of mapping classes is either reducible, finite, or fixes one of the attractors. In particular, not every braid can arise. The proof is based on the finiteness of the modular group for a connected component of the Julia set, discussed in [Mc2].

This leads to a topological criterion for the existence of bifurcations in iterative root-finding algorithms based on rational maps (such as Newton's method). For example, any such algorithm must fail on every neighborhood of $p(X)=X^{d}$ when $d$ is 4 or more. We show that on such neighborhoods one cannot even continuously assign to each polynomial $p$ an expanding rational map $T_{p}$ such that the attractor of $T_{p}$ is contained in the roots of $p$.

\section{Outline of the paper}

$\$ \S 1$ and 2 present our results on the failure of algorithms and braiding of the attractor. These are really two formulations of the same result; in $\S 2$ the former is deduced from the latter. $\$ \$ 3$ and 4 present the proofs. First we show that topological assumptions on the braiding of the attractor imply the existence of a separator, that is a characteristic component of the Julia set which separates the attracting periodic points from one another. Then the results of [Mc2] imply the monodromy of this component (by holomorphic motions) is a finite group, completing the proof in $\$ 4$.

In the Appendix we give an example of a stable family in which the braiding is pseudo-Anosov.

* Research partially supported by NSF Grant 8120790 


\section{§1. Iterative root-finding algorithms}

We begin with some terminology of Smale's $[\mathrm{Sm}]$.

Definitions. A purely iterative algorithm is a mapping

$$
T: \text { Poly }_{d} \rightarrow \text { Rat }_{k}
$$

associating to each degree $d$ complex monic polynomial $p(X) \in \operatorname{Poly}_{d}\left(\cong \mathbf{C}^{d}\right)$ a degree $k$ rational function $T_{p}(z) \in \operatorname{Rat}_{k}\left(\cong \mathbf{P}^{2 k+1}\right)$, such that the coefficients of $T_{p}$ are themselves rational functions of the coefficients of $p$. The algorithm is said to be generally convergent if there is an open dense set of full measure $U \subset$ Poly $_{d} \times \mathbf{C}$ such that under iteration,

$$
T_{p}^{n}(z) \rightarrow\{\text { a root of } p\}
$$

for all choices of polynomial and initial guess $(p, z)$ in $U$.

We call a generally convergent algorithm robust if the image of $T$ is contained in $\operatorname{Exp}_{k}$, the open subset of $\mathrm{Rat}_{k}$ consisting of expanding rational maps. Here a rational map is expanding if its dynamics satisfies Smale's Axiom $A$. Equivalently, $\operatorname{Exp}_{k}$ consists of those rational maps whose critical points all tend toward attracting cycles under iteration. (See [Su11] for a discussion of these maps.)

Robustness insures good global behavior: for any neighborhood $U$ of the roots of $p$, the spherical area of the set of initial guesses which take more than $n$ iterates to land in $U$ tends to zero goemetrically fast (it is $O\left(\lambda^{n}\right)$ for some $\lambda<1$.)

\section{Examples}

(1) Newton's method is a purely iterative algorithm for each degree $d$, and it is generally convergent for $d=2$ but not 3 or more.

(2) There exists a generally convergent algorithm for cubics; for

$$
p(X)=X^{3}+a X+b
$$

take $T_{p}(z)$ to be Newton's method applied to the rational function

$$
r(X)=\frac{X^{3}+a X+b}{3 a X^{2}+9 b X-a^{2}} .
$$

Newton's for quadratics and the above method for cubics are both robust.

In [Mc1] we classify generally convergent algorithms and answer a question of Smale's by proving:

Theorem 1.1. There is no generally convergent purely iterative algorithm for finding the roots of polynomials of degree 4 or more.

The proof of Theorem 1.1 relies strongly upon the algebraic character of the family $T_{p}$, and being a proof by contradiction it does not seem to reveal where algorithms for degrees 4 or more fail. 
Here we develop a localized criterion for the failure of algorithms. We will show, for example, that every purely iterative algorithm must fail on every neighborhood of $p(X)=X^{d}$ for $d=4$ or more. As far as robust algorithms are concerned, when the criterion is satisfied one cannot even continuously assign to each polynomial $p$ an expanding map $T_{p}$ such that the attractor of $T_{p}$ is contained in the roots of $p$.

The criterion is defined in terms of the braiding of the roots of polynomials as we move about the Poly ${ }_{d}$; a good introduction to these ideas is Birman's book [Bi]. We also use the concept of 'reducibility' of an element or subgroup of the mapping class group; this originates with Nielsen's notion of 'simple axis' and is fundamental to Thurston's classification of elements [FLP].

Notation. For $A$ any finite subset of $\widehat{\mathbb{C}}, \operatorname{Mod}(A)$ will denote the full mapping class group of the surface $\hat{\mathbb{C}}-A$. (We include maps which do not fix $A$, but we exclude orientation-reversing maps. Our notation comes from $\operatorname{Mod}(A)$ 's role as the 'modular group' for Teichmüller space.)

Let $\Delta$ denote the discriminant hypersurface in Poly $_{d}$, i.e. the locus of polynomials with at least one multiple root. Let $(\gamma, p)$ be a closed loop in the complement of $\Delta$ with basepoint $p(X)$, and let $r$ be the set of roots of $p$. As we traverse $\gamma$ the roots of the corresponding polynomials move without collision, and hence their monodromy determines an element in $\operatorname{Mod}(r)$, the full mapping class group of the Riemmann sphere $\widehat{\mathbb{C}}$ punctured at $r$. There is a natural homomorphism $\operatorname{Mod}(r) \rightarrow \operatorname{Sym}(r)$, the group of permutations of the set of roots, such that the image of $\gamma$ in $\operatorname{Sym}(r)$ records how the roots are interchanged by the monodromy.

Now let $V$ be an open connected subset of Poly ${ }_{d}$, and let $p$ be a polynomial in $p$ with simple roots $r$. Then there are homomorphisms

$$
\pi_{1}(V-\Delta, p) \rightarrow \operatorname{Mod}(r) \rightarrow \operatorname{Sym}(r)
$$

with images $G_{\text {top }}$ and $G_{\text {comb }}$ respectively, recording the topological and combinatorial monodromy of the roots of $p$ over $V$.

Definition. $V$ is tangled if the following three conditions are satisfied:

(1) $G_{\text {comb }}$ acts transitively on the roots $r$;

(2) $G_{\text {top }}$ is irreducible; and

(3) $G_{\text {top }}$ is infinite.

Here a subgroup of the mapping class group of a surface $S$ is irreducible if it preserves no system of disjoint nonperipheral simple closed curves on $S$. (Finite irreducible subgroups are discussed by Gilman [G]).

Conditions 2) and 3) are implied by the (perhaps more easily verified) combinatorial conditions

(2') $G_{\text {comb }}$ is irreducible;

(3') $G_{\text {comb }}$ is not isomorphic to a finite group of Möbius transformations (a cyclic group $\mathbf{Z} / n$, a dihedral group $D_{n}$, the alternating group $A_{4}$, the octahedral group, or the icosahedral group.)

Here a subgroup of $\operatorname{Sym}(r)$ is irreducible if it preserves no nontrivial partition of the set $r$. (Transitivity does not imply irreducibility: e.g. (1234) preserves 
the partition $\{[1,3],[2,4]\}$.) If $G_{\text {top }}$ is reducible, an invariant partition can be constructed using the 'innermost' simple closed curves, so $\left(2^{\prime}\right) \Rightarrow(2)$.

The implication $\left(3^{\prime}\right) \Rightarrow(3)$ follows from Nielsen realization: any finite subgroup of the modular group of a multiply-punctured sphere can be realized by a group of Möbius transformations after appropriately relocating the punctures. (See Zieschang, [Z, 54.3] where the Nielsen problem is solved for surfaces with punctures; the general case was later proved by Kerckhoff [Ker]).

Examples. Assume $d$ is 4 or more. The following sets are tangled:

(a) Any open connected neighborhood of $p(X)=X^{d}$.

(b) Any $V$ whose monodromy permutes roots arbitrarily $\left(G_{\text {comb }}=\operatorname{Sym}(r)\right)$. (This includes (a) as a special case.)

(c) Any neighborhod of a loop in the complement of the discriminant locus, such that the monodromy around the loop is transitive on the roots and pseudoAnosov on their complement.

Theorem 1.2 (Location of Failures). Let $V \subset$ Poly $_{d}$ be open, connected and tangled. Then

(a) Any purely iterative algorithm fails on $V$.

(b) There is no continuous map $T: V \rightarrow \operatorname{Exp}_{k}$ such that the attractor of $T_{p}$ is contained in the roots of $p$ for each $p$ inV.

In part (a), by 'fail' we mean there exists an open set $U$ in $V \times \mathbf{C}$ such that $z$ is a bad initial guess for $T_{p}$ for every $(p, z)$ in $U$.

\section{Complements}

(1) Since the notion of tangledness depends only on monodromy around loops, part (b) of the Theorem gives a topological obstruction to the construction of a robust algorithm over the 1 -skeleton of Poly $d-\Delta$ for $d \geqq 4$. One can ask if there is already an obstruction over the 0-skeleton: does there exist a finite set $A \subset \mathbb{C}$ which is not the attractor of any rational map? If so, the polynomial with roots at $A$ would be 'universally unsolvable' by rational iteration.

In fact, by a recent result of Flexor and Sentenac [FS], there is no universally unsolvable polynomial: for any $p(z)$, one can choose a complex number $h$ such that modified Newton's method

$$
N_{h}(z)=z-h \frac{p(z)}{p^{\prime}(z)}
$$

is an expanding rational map whose attractor coincides with the roots of $p$. However, by Theorem 2.1(b), $h$ cannot be chosen continuously over any tangled set of $p$ 's!

(2) Shub and Smale have shown [SS] that if one allows complex conjugation in the formula for $T_{p}(z)$ (so the resulting map is only real-algebraic) then there exist generally convergent algorithms for all degrees.

(3) One can remain in the complex algebraic category and consider, more generally, finite towers of purely iterative algorithms. In this context polynomials of degree 5 become solvable but those of degree 6 are not [DM]. 
(4) Although Theorem 1.2 implies 1.1, the techniques we use here do not yield the rigidity result for algebraic families which underlies the proof of Theorem 1.1 in [Mc1]. Similarly, the statement of Theorem 1.1 in [Mc1] is formally stronger than that given here: the 'full measure' condition is dropped from the definition of general convergence. Here we need that condition to rule out invariant Beltrami differentials supported on the Julia set.

\section{§. Braiding of the attractor}

Since a $d$-stranded braid is the same as an injective motion of $d$ points on the sphere, every braid can be realized as the motion of roots over a loop in Poly $_{d}-\Delta$. If we follow the attractor of a rational map as we move about in a stable family, it too describes a braid, but not every braid can arise (Theorem 2.1; see Fig. 2.1 for an example). The definition of a tangled subset of Poly insures that the roots are braided too generally to coincide with the braiding of the attractor of a rational map.

Definitions. A family of rational maps $f_{\lambda}(z)$ is holomorphic if the coefficients of $f$ are holomorphic functions of the parameter $\lambda$; it is stable if the maps all have the same degree and there is a uniform upper bound on the periods of attracting cycles for maps occurring in the family. Stability rules out bifurcations, and maps in the same component of the parameter space are quasiconformally conjugate on their Julia sets [MSS].

Let $(X, f)$ be a stable holomorphic family of rational maps parameterized by a connected complex manifold $X$ with basepoint representing the rational map $f(z)$. We say $f$ is attractive if there is an open dense set of full measure $U \subset \hat{\mathbb{C}}$ and a finite set $A$ such that $f^{n}(z)$ tends to $A$ for all $z$ in $U$. (By stability, if this condition is true for $f$ is true for every other map in the family.) We call $A$ the attractor of $f$ and $(X, f)$ an attractive family.

Our condition on $f$ is equivalent to the condition: $f(z)$ has no Siegel disks or Herman rings and the Julia set of $f$ has zero area.

The attractor $A$ is just the union of the attractive, superattractive and rationally indifferent periodic cycles for such an $f$.

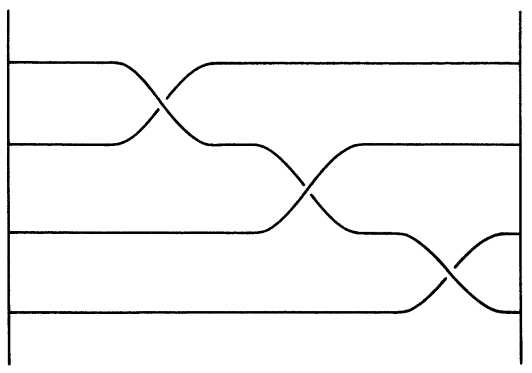

Fig. 2.1. This braid does not arise for rational maps 
A good example to keep in mind is that of an expanding map $f(z)$, and $X$ the open component of $\operatorname{Exp}_{k}$ containing $f$. Another example is the pair $\left(\right.$ Poly $\left._{d}, T_{p}\right)$, where $T_{p}$ is any generally convergent algorithm. (If $T_{p}$ is not a stable family, then there exist attracting periodic cycles of arbitrarily high order for certain values of $p$; these persist locally so $T_{p}$ is not generally convergent.)

As we move about in $X$, the periodic cycles of $f$ move injectively; in particular, the monodromy of the motion of $A$ determines a homomorphism

$$
\pi_{1}(X, f) \rightarrow \operatorname{Mod}(A)
$$

whose image $G$ records the braiding of the attractor. We refer to $G$ as the monodromy group of the family $(X, f)$.

Theorem 2.1 (Controlled braiding). For any attractive family, the monodromy group $G$ is either (a) finite, (b) reducible, or (c) fixes a point of $A$.

Remark. There exists an attractive family of polynomials with $|A|=4$, such that $G$ contains a pseudo-Anosov element which fixes infinity and cyclically permutes the three finite attractors (see the Appendix). Thus in case (c) $G$ can be infinite irreducible.

Proof of Theorem 1.2 (Location of failures). Let $V$ be a tangled subset of Poly ${ }_{d}$. We begin by proving any purely iterative algorithm $T_{p}$ fails on $V$. We may assume that $V$ meets neither the discriminant locus nor the (possibly empty) subvariety on which $T_{p}$ is undefined or represents a rational map of degree less than $k$. If $T_{p}$ does not fail on $V$, then $\left(V, T_{p}\right)$ is an attractive family, with attractor $A$ contained in $r$, the roots of $p$.

As before, let $G_{\text {top }}$ denote the topological monodromy of the roots over $V$, and let $G \subset \operatorname{Mod}(A)$ record the braiding of the attractor. Since for each $q$ in $V$, the attractor of $T_{q}$ is contained in the roots of $q, G$ is the image of $G_{\text {top }}$ induced by the inclusion $A \subset r$.

Since $V$ is tangled, $G_{\text {top }}$ is transitive, irreducible and infinite. Transitivity implies that $A=r$ and $G=G_{\text {top }}$. But then $G$ violates all three possibilities enumerated in Theorem 2.1, a contradiction. Thus $T_{p}$ must fail on $V$, establishing part (a) of the theorem.

Now suppose the family $\left(V, T_{p}\right)$ consists of expanding maps, varying only continuously with $p$, such that for each $q$ in $V$ the attractor of $T_{q}$ is contained in the roots of $q$. Let $X$ denote the component of $\operatorname{Exp}_{k}$ containing $T_{p}$. The map $T: V \rightarrow \operatorname{Exp}_{k}$ induces a map

$$
\pi_{1}\left(V, T_{p}\right) \rightarrow \pi_{1}\left(X, T_{p}\right) \rightarrow \operatorname{Mod}(A)
$$

exhibiting, as before, the image of $G_{\text {top }}$ under the inclusion $A \subset r$ as a subgroup of $G$, the monodromy of the attractor in the attractive family $\left(X, T_{p}\right)$. Again it follows that $A=r$ and $G$ is transitive, irreducible and infinite, contradicting Theorem 2.1 and so establishing part (b). 


\section{§3. Partitions of the sphere}

This section and the next are devoted to the proof of Theorem 2.1.

Here we present a topological criterion for certain partitions of the sphere to contain a component $K$ which completely separates the elements of a finite set $A$ from one another.

The next section begins with the definition of the modular group of a rational map and its relation to the monodromy group $G$ in an attractive family. Then assuming $G$ is irreducible and fixed-point free, the criterion of this section gives a component $K$ of the Julia set which separates the points of the attractor $A$. Finally appeal to the main result of $[\mathrm{Mc} 2]$ (that $\operatorname{Mod}(K, f)$ is a finite group) shows $G$ is finite, completing the proof.

Definitions. Let $A$ and $K$ be subsets of $\hat{\mathbb{C}}$, with $A$ finite. Assume $K$ is connected and either open or closed. (In applications the set $A$ will be the attractor of a rational map, and $K$ will be a component of the Julia set or its complement.) Let $\left\langle D_{i}, i=1, \ldots, n\right\rangle$ denote the components of $\hat{\mathbb{C}}-K$ which meet $A$.

We define an invariant $\Gamma(K)$ which records the separation properties of $K$ relative to $A$. Begin by constructing a system $\left\langle\gamma_{1}, \ldots, \gamma_{n}\right\rangle$ of disjoint simple closed curves such that $\gamma_{i}$ separates $A \cap D_{i}$ from the remaining points of $A$. If $K$ is open, we require that these curves lie in $K$, while if $K$ is closed we require they lie in $\hat{\mathbb{C}}-K$.

Define $\Gamma(K)$ as the set of free homotopy classes on $\hat{\mathbb{C}}-A$ represented by $\left\langle\gamma_{1}, \ldots, \gamma_{n}\right\rangle$. By convention, we also include in $\Gamma(K)$ a peripheral loop around each element of $A \cap K$. These free homotopy classes depend only on $A$ and $K$ (see Figure 3.1 for an example with $K$ closed).

Remarks. (1) If $\phi$ is a homeomorphism of $(\hat{\mathbb{C}}, A)$, then $\Gamma(\phi(K))=\phi(\Gamma(K))$ (the construction is natural).

(2) If $K$ and $L$ are disjoint, the union of $\Gamma(K)$ and $\Gamma(L)$ can be represented by simple closed curves. The same is true for any finite collection of components.

Now let $J$ be a closed subset of $\hat{\mathbb{C}}$ (in applications $J$ will be the Julia set of a rational map) Let part $(J)$ denote the set of components of $J$ union the set of components of $\hat{\mathbb{C}}-J$. Then $\operatorname{part}(J)$ is a partition of the Riemann sphere into sets $K$ each of which is connected and either open or closed.

Definition. A separator $K$ is an element of part $(J)$ such that $\Gamma(K)$ consists of a peripheral curve around each point of $A$. (Equivalently, the points of $A$ not lying in $K$ lie in distinct components of $\hat{\mathbb{C}}-K$.)

Theorem 3.1. Let $J$ and $A$ be subsets of $\widehat{\mathbb{C}}$ with $J$ closed and $A$ finite. Suppose $\Gamma(K)$ is consists only of peripheral curves for all $K$ in $\operatorname{part}(J)$. Then part $(J)$ contains a separator.

Proof. Assume $|A| \geqq 3$ (otherwise any element of part $(J)$ containing a point of $A$ is a separator). Then any peripheral curve encircles a unique point in $A$; let $A(K)$ denote the points encircled by $\Gamma(K)$.

There are three possibilities for $A(K): A(K)=A, A(K)=\emptyset$, or $A(K)=\{a\}$ for some point of $A$. (This trichotomy holds for any $K$ with $\Gamma(K)$ totally peripheral.) If $A(K)=A, K$ is the sought-after separator. 


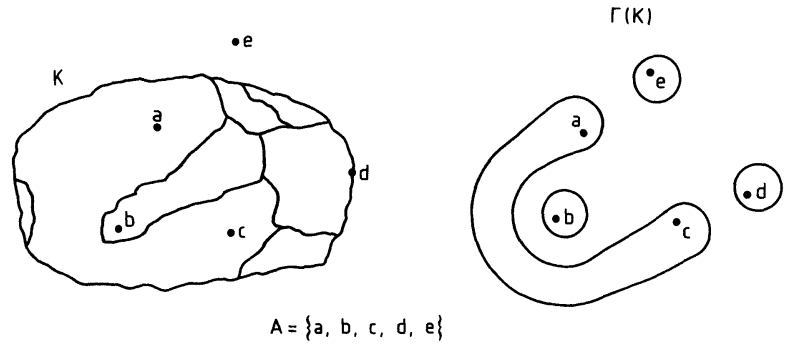

Fig. 3.1 Construction of $\Gamma(K)$

So assume $A(K)$ is either empty or a singleton for all components $K$; we will deduce a contradiction.

First we simplify the partition to eliminate elements with $A(K)$ empty. Define

$$
\text { fill }(K)=\hat{\mathbb{C}}-U\{\text { components of } \hat{\mathbb{C}}-K \text { which meet } A\} ;
$$

i.e. fill $(K)$ is obtained from $K$ by filling in the components of its complement which do not meet $A$. Using plane topology (e.g. $[W]$ ), one may check that

fillpart $(J)=\{$ fill $(K): K$ is an element of $\operatorname{part}(K)$ with $A(K)$ nonempty $\}$

is again a partition of the Riemann sphere into closed and open sets, and $A(K)$ is a singleton for every element of fillpart $(J)$.

Let $p: \hat{\mathbb{C}} \rightarrow A$ map each $K$ in fillpart $(J)$ to the singleton in $A(K)$. It is straightforward to verify that $p$ is continuous and surjective, contradicting the connectivity of $\hat{\mathbb{C}}$. Therefore part $(J)$ must contain a separator.

\section{§4. Locating a separator in the Julia set}

Definitions. Let $f$ be an attractive map with Julia set $J$ and attractor $A$.

If $E$ is closed subset of $\hat{\mathbb{C}}$ such that $f(E)=E$, then $\operatorname{Mod}(E, f)$, the modular group of $f$ on $E[\mathrm{Mc} 2, \S 3]$, is the group of equivalence classes [ $\phi]$ of maps $\hat{\mathbb{C}} \rightarrow \widehat{\mathbb{C}}$ such that

(1) $\phi$ is quasiconformal;

(2) $\phi(E)=E$; and

(3) $\phi \circ f(z)=f \circ \phi(z)$ for all $z$ in $E$.

By definition, two maps $\phi$ and $\psi$ are equivalent iff they can be connected by an isotopy through homeomorphism enjoying (1-3).

Remarks. We will be mostly concerned with the case $E=J \cup A$. For $[\phi]$ in $\operatorname{Mod}(J \cup A, f), \phi$ behaves much like a global conjugacy. For example:

(1) $[\phi]$ determines a well-defined map $\phi \mid J \cup A$ (since periodic points are dense in $J \cup A$, the values of this map cannot change during isotopy). Similarly, if $U$ is a component of $\hat{\mathbb{C}}-J \cup A$, the image component $\phi(U)$ is well-defined, and $\phi$ commutes with the action of $f$ on components. 
(2) If $z$ is an attracting or superattracting periodic point for $f$, then so is $\phi(z)$ (since these are the only isolated point of $J \cup A$ ).

(3) If $z \in J$ is a critical point (repelling periodic point, indifferent periodic point with multiplier $\left.e^{i \theta}\right)$, then the same is true of $\phi(z)$.

In particular, $\phi(A)=A$ for any $[\phi]$ in $\operatorname{Mod}(J \cup A, f)$.

Proof of (3). The Julia set is perfect and totally invariant, so points in $J$ are mapped locally $n$-to-1 near $z$ iff $z$ is a critical point of order $(n-1)$ (and the former property is preserved by a topological conjugacy on $J$.)

To handle periodic points we pass to microscopic scale and take a limit. Let $z \in J$ be a periodic point, which we may asume to be a fixed point, and let $\alpha$ and $\beta$ denote the derivatives of $f$ at $z$ and $\phi(z)$ respectively. Choose points $w_{n} \in J$ such that $w_{n} \rightarrow z$, and let $A_{n}, B_{n}$ be the Möbius transformations which carry $\left(z, w_{0}, w_{n}\right)$, and $\left(\phi(z), \phi\left(w_{0}\right), \phi\left(w_{n}\right)\right)$ to $(0, \infty, 1)$. Then $B_{n} \circ \phi \circ A_{n}^{-1}$ is a sequence of normalized quasiconformal mappings which are conjugacies on $A_{n}(J)$. After passing to a subsequence these converge to a quasiconformal map $\psi$ which is a conjugacy between $z \rightarrow \alpha z$ and $z \rightarrow \beta z$ on $J^{\prime}$, a Hausdorff limit of $A_{n}(J)$ (containing 1 and invariant under multiplication by $\alpha$ ).

Since $\psi(1)=1, \alpha^{n} \rightarrow \infty$ if and only if $\beta^{n} \rightarrow \infty$, establishing (3) for repelling periodic points. If $\alpha$ is not a root of unity, then $\left\langle\alpha^{n}\right\rangle$ is dense in the unit circle and we easily conclude $\alpha=\beta$. Finally if $\alpha$ is a primitive $n$th root of unity, so is $\beta$. Using the 'Fatou flower theorem' ( $[\mathrm{Bl}, 3.12]$ and references therein), we conclude that $J^{\prime}$ contains rays emanating from the origin and passing through the powers of $\alpha$. Since $\phi$ is a homeomorphism, the order relation of these rays is preserved and $\alpha=\beta$.

Definition. The universal monodromy group of $(A, f)$ is the image of the natural map

$$
\operatorname{Mod}(J \cup A, f) \rightarrow \operatorname{Mod}(A)
$$

We will denote this group by $\operatorname{Univ}(A, f)$.

Proposition 4.1. The monodromy group $G$ for an attractive family $(X, f)$ is a subgroup of the universal monodromy group $\operatorname{Univ}(A, f)$.

Proof. The motion of the periodic points determines a conjugating isotopy of $J \cup A$ which can be extended quasiconformally to the whole Riemann sphere [MSS, ST, BR]. Thus the monodromy of the attractor factors through the modular group of $f$ on $J \cup A[\mathrm{Mc} 2,3.2]$ and consequently through the universal monodromy group of $(A, f)$ :

$$
\pi_{1}(X, f) \rightarrow \operatorname{Mod}(J \cup A, f) \rightarrow \operatorname{Univ}(A, f) \subset \operatorname{Mod}(A) .
$$

By the above proposition, $\operatorname{Univ}(A, f)$ is irreducible and fixed-point free if there exists a single attractive family $(X, f)$ whose monodromy has this property. For the remainder of the discussion we will concentrate on proving that this property implies $\operatorname{Univ}(A, f)$ is finite; by the above, this will establish Theorem 2.1.

Warning. We do not guarantee that every element of $\operatorname{Univ}(A, f)$ can be realized by the monodromy in an attractive family including $f$. 
Adopting the notation of the preceding section, we now observe:

Proposition 4.2. If $\operatorname{Univ}(A, f)$ is irreducible, then part $(J)$ contains a separator $K$.

Proof. Let $\Gamma$ denote the union of the free homotopy classes $\Gamma(K)$ over all elements $K$ of $\operatorname{part}(J)$. Since any two elements of part $(J)$ are disjoint, $\Gamma$ can be represented by a finite collection of disjoint simple closed curves on $\hat{\mathbb{C}}-A$. Every $[\phi]$ in $\operatorname{Mod}(J, f)$ maps $J$ to $J$, so the system of curves $\Gamma$ is preserved (up to homotopy) by $\operatorname{Univ}(A, f)$. (Cf. the Remarks in $\S 3)$.

If $\operatorname{Univ}(A, f)$ is irreducible, $\Gamma$ (and hence each $\Gamma(K)$ ) contains only peripheral curves. Then by Proposition 3.1, part $(J)$ contains a separator.

Proposition 4.3. Suppose $|A| \geqq 3$, $\operatorname{Univ}(A, f)$ fixes no point of $A$, and $K$ in part $(J)$ is a separator. Then

(a) $K$ is a component of the Julia set, and

(b) $f(K)=K$.

Proof. Call a set $U \subset \hat{\mathbb{C}}$ distinguished if $\phi(U)=U$ for all $\phi$ in $\operatorname{Mod}(J \cup A, f)$. By hypothesis, no point of $A$ is distinguished.

We claim there is no distinguished component of $\hat{\mathbb{C}}-J$. Indeed, if $U$ is a component of $\hat{\mathbb{C}}-J$, there is a $k$ such that $f^{k}(U)$ coincides with the immediate basin of attraction of some point $\{a\}$ in $A$ (since $f$ is attractive), and if $U$ were distinguished $\{a\}$ would be too. The proofs of (a) and (b) rely on this fact.

(a) $|A| \geqq 3$ implies $K$ is the unique separator in $\operatorname{part}(J)$. Thus $K$ is distinguished. Since there is no distinguished component of $\widehat{\mathbb{C}}-J, K$ is a component of $J$.

(b) Let $B$ denote the union of the immediate basins of attraction of the points in $A$. $B$ is a finite union of open components of $\widehat{\mathbb{C}}-J$, so the same is true of $f^{-n}(B)$ for each $n$. We have

$$
B \subset f^{-1}(B) \subset f^{-2}(B) \ldots \text { and } \cup f^{-n}(B)=\hat{\mathbb{C}}-J .
$$

For each $n$, let $F_{n}$ denote the component of the complement of $f^{-n}(B)$ which contains the separator $K$. Then $\cap F_{n}=K$. If $f\left(F_{n+1}\right) \subset F_{n}$ for all $n$, then $f(K)=K$ as claimed.

Otherwise there exists a least $n$ such that $f\left(F_{n+1}\right) \cap F_{n}=\emptyset$. To complete the proof, we will use this situation to produce a distinguished component $U$ in $\widehat{\mathbb{C}}-J$.

Since $K$ is distinguished, so are $F_{n}$ and $f\left(F_{n+1}\right)$. There is a unique component $V$ of $\hat{\mathbb{C}}-F_{n}$ which contains $f\left(F_{n+1}\right) . V$ is an open disk, and $\partial V \subset F_{n}$, so $V \cap f^{-n}(B)$ consists of a finite number of components of $f^{-n}(B)$; among these there is a unique component $U$ such that $\partial V \subset \partial U$. $U$ is constructed naturally from distinguished objects, so $U$ itself is distinguished. This is the desired contradiction.

The final idea in the proof is that the separator provides a collar constraining the motion of the attractor.

Proposition 4.4 If $\operatorname{Univ}(A, f)$ is irreducible and fixed-point free, then it is a finite group. 
Proof. We may assume $|A| \geqq 4$ (otherwise $\operatorname{Mod}(A)$ itself is finite and there is nothing to prove). Propositions 4.2 and 4.3 imply the existence of a separator $K$ such that $f(K)=K$. $K$ is a distinguished component of the Julia set, so the map from $\operatorname{Mod}(J \cup A, f)$ onto $\operatorname{Univ}(A, f)$ can be factored:

$$
\operatorname{Mod}(J \cup A) \rightarrow \operatorname{Mod}(K \cup A, f) \rightarrow \operatorname{Univ}(A, f) .
$$

By the definition of an attractive map, $K$ has measure zero. Thus $K$ carries no invariant line field and by $[\operatorname{Mc} 2,3.4], \operatorname{Mod}(K, f)$ is a finite group. (A sketch of the argument: one may perform surgery to create a new rational map $g$ for which elements of $\operatorname{Mod}(K, f)$ can be realized as conformal automorphisms - i.e. Möbius transformations commuting with $g$. And the group of such automorphisms is finite.)

Since $K$ is a separator, the natural homomorphism $\operatorname{Mod}(K \cup$ $A, f) \rightarrow \operatorname{Mod}(K, f)$ is injective. (Each component of $\widehat{\mathbb{C}}-K$ is a disk containing at most one point of $A$. If $[\phi]$ in $\operatorname{Mod}(K \cup A, f)$ is isotopic to the identity rel $K$, the isotopy can be easily modified so it is also rel $A$.) Hence $\operatorname{Mod}(K \cup A, f)$ and $\operatorname{Univ}(A, f)$ are both finite.

Proof of Theorem 2.1 (Controlled Braiding). Let $(X, f)$ be an attractive family with monodromy $G$; by Proposition $4.1 G$ is a subgroup of $\operatorname{Univ}(A, f)$. If $G$ is irreducible and fixed-point free, the same is true of $\operatorname{Univ}(A, f)$; then by the above $\operatorname{Univ}(A, f)$ is finite, so $G$ is finite.

Acknowledgements. I'd like to thank Peter Doyle and Jane Gilman for discussions and for suggesting improvements.

\section{Appendix: A stable family with pseudo-Anosov monodromy}

Our construction begins with a function $f(z)$ with the following properties.

1) The map $f(z)$ is a degree 6 monic polynomial, with three finite simple critical points $c_{1}, c_{2}, c_{3}$, such that $f\left(c_{i}\right)=c_{i}$; and a fourth critical point $x$ of multiplicity two such that $f^{n}(x) \rightarrow \infty$.

2) The leaf through $x$ of the canonical foliation of the basin of $\infty$ is a bouquet of three circles, each enclosing one of the $c_{1}$ and mapped by degre 2 to the leaf through $f(x)$. (The foliation we refer to is the closure of the small orbit relation; see $[\mathrm{Bl}, \S 9]$ or [Sul2].)

It follows that from (1) that $f$ is expanding, with attractor $A=\left\{c_{1}, c_{2}, c_{3}, \infty\right\}$.

For $X$ we take the component of the space of expanding degree 6 polynomials which contains $f$. Then $(X, f)$ is an attractive family.

Theorem A.1. The monodromy group $G$ of $(X, f)$ contains a pseudo-Anosov transformation fixing $\infty$ and cyclically permuting the other three points in the attractor.

The proof will use only properties (1) and (2), so we first discuss the existence of such an $f$.

Proposition A.2. There exists a polynomial $f(z)$ satisfying (1) and (2).

Proof. This can be verified by an algebraic calculation, or done quasiconformally. The latter approach is more general and conceptual; to describe it, we introduce some notation: $L_{n}$ will denote the leaf of the foliation through $f^{n}(x), A_{n}$ the annulus bounded by $L_{n}$ and $L_{n+1}$. Note that $L_{0}$ is the bouquet through $x$ (Fig. A.1).

We will build a smooth map with the desired dynamics, then observe it is conjugate to a rational map.

Begin by picking points to be $\left\{c_{1}, c_{2}, c_{3}, f(x)\right.$ and $\left.f^{2}(x)\right\}$, and drawing real analytic curves to represent the leaves $L_{i}, i=0,1,2$, arranging the petals of the bouquet to meet in $60^{\circ}$ angles at $x$. Map the disks enclosed by each petal conformally by degree 2 to the disk enclosed by the 
leaf through $f(x)$, arranging the critical point and value both to be equal to $C_{2}^{\prime}$, and of course sending $x$ to $f(x)$. Now map the region outside the leaf through $f(x)$ conformally by degree 6 to the region outside the leaf through $f^{2}(x)$, arranging that $\infty$ is both the critical point and critical value for this map. Finally map the annulus $A_{0}$ between the bouquet and $L_{1}$ smoothly to the annulus $A_{1}$, so that the map fits together with that already defined and creates a branch point at $x$.

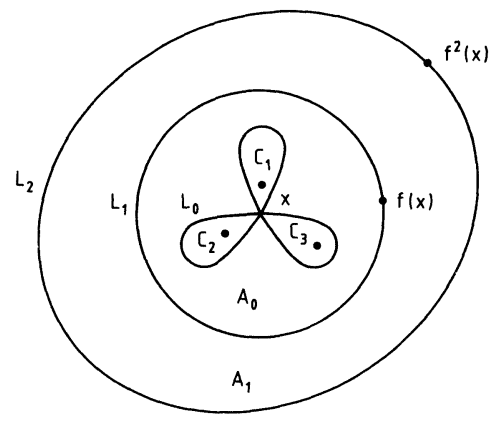

Fig. A.1. Leaves of the canonical foliation for $f(x)$

This defines a smooth branched cover of the sphere. We claim its iterates are uniformly quasiconformal. Indeed, the map is conformal except on the annulus $A_{0}$; but under iteration the points in $A_{0}$ never return to there (they tend to $\infty$ ), so the dilatation of the iterates is bounded by that of the original map.

By constructing an invariant Beltrami differential and using the measurable Riemann mapping theorem as in [Sul2, Theorem 9], we conclude that this smooth map is quasiconformally conjugate to a rational map $f(z)$ with properties (1) and (2).

Our next task is to produce closed loops in $X$ with prescribed monodromy. Let $\operatorname{Mod}(f)$ denote the group of isotopy classes of quasiconformal maps $\phi$ such that $\phi(f(z))=f(\phi(z))$ on the entire Riemann sphere. (This group is discussed in more detail in $[\mathrm{Mc} 2, \S 2]$.) Since the attractor is topologically characterized, $\phi(A)=A$ for $[\phi]$ in $\operatorname{Mod}(f)$.

Proposition A.3. The monodromy group of $(X, f)$ contains the image of $\operatorname{Mod}(f)$ in $\operatorname{Mod}(A)$.

Proof. The argument, which is very general, comes from [Sul2]. Let $\mu$ denote the Beltrami differential of $\phi$ and for each $t$ in $[0,1]$ form the map $f_{t}=\phi_{t}^{-1} \circ f_{\circ} \phi_{t}$, where $\phi_{t}$ is the unique quasiconformal map with dilatation $t \mu$ agreeing with $\phi$ at $\infty$ and two other points. This exhibits a path in $X$ connecting $f$ to a polynomial conjugate to $f$ by a Möbius transformation $M$. By connecting $M$ by a path to the identity, we obtain a loop with the desired monodromy.

Proposition A.4. The monodromy group $G$ contains a element of order 3 , fixing $\infty$ and cyclically permuting the three finite points of the attractor.

Proof. By the preceding, it is enough to construct a map in $\operatorname{Mod}(f)$ with this property. Begin with a quasiconformal map $\phi$ of $A_{0}$ to itself which is the identity on the outer boundary and cyclically permutes the inner petals, fixing $x$. The map on the petals is determined by requiring $\phi$ to commute with $f$ on the boundary of $A_{0}$.

An appropriately chosen $\phi$ can be extended to a map commuting with $f$ on the whole Riemann sphere. The requirement is that $\phi$ respect the rigid structure on each leaf of the foliation in $A_{0}$. Then the dynamics can be used push $\phi$ forward to the successive annuli $A_{n}$ filling in a neighborhood of infinity. On the remainder of the basin of $\infty f$ is a covering, and can be used to pull $\phi$ back. Finally we fill $\phi$ in conformally on the remainder of the sphere. This is possible because the dynamics is conformally the same on the immediate basin of each $c_{i}$, and the pre-images of the immediate basins are all disks. 
It is not hard to see $\phi$ is a homeomorphism commuting with $f$ and quasiconformal except possibly on the Julia set. Quasiconformality on the Julia set follows from the expanding property of $f$ [Sul2].

By construction, $\phi$ fixes $\infty$ and cyclically permutes the three finite points in the attractor. The map $\phi^{3}$ fixes the petals, and hence is isotopic to the identity on $\widehat{\mathbb{C}}-A$.

Proposition A.5. The monodromy group $G$ contains a half Dehn twist about a simple closed curve separating two points in the attractor from the two others.

Proof. Since $X$ is an open subset of Poly ${ }_{d}$, we may perturb $f$ slightly to a new map $g$ in $X$, such that the critical point $x$ splits into two critical points $y$ and $w$, still attracted to infinity but lying on different leaves of the foliation (Fig. A.2). One of the points, say $y$, lies on the innermost leaf of the two. As in the preceding proof, we can construct a map $\phi$ in $\operatorname{Mod}(g)$ which is the identity on the leaf through $w$ and interchanges the two petals on the leaf through $y$. This map is a half twist on the annulus bounded by these two leaves, and hence its mapping class is represented by a half twist about the core curve of this annulus.

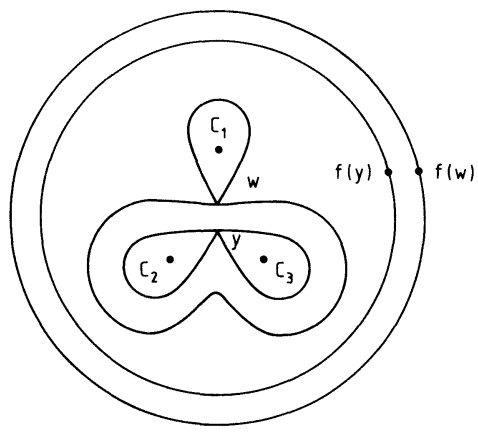

Fig. A.2. The foliations of the perturbed map $g$

By Proposition A.3 there is a loop in $X$ based at $g$ inducing this monodromy. Joining this loop to $f$ by a path only changes the monodromy by an inner automorphism of $\operatorname{Mod}(A)$, so there is an element of $G$ with the desired properties.

Completion of the Proof of Theorem A.1. Conjugating the half twist by the element of order 3, we obtain two half twists about intersecting simple closed curves. The product of one with the inverse of the other is pseudo-Anosov and cyclically permutes the finite points of $A$.

\section{References}

[BR] Bers, L., Royden, H.: Holomorphic families of injections. Acta Math. 157, 259-286 (1986)

[Bi] Birman, J.: Braids, Links and Mapping Class Groups. Ann Math. Stud. 82, Princeton, 1974

[B1] Blanchard, P.: Complex analytic dynamics on the Riemann sphere. Bull. AMS 11, 85-141 (1984)

[DM] Doyle, P., McMullen, C.: Solving the quintic by iteration (In preparation)

[FLP] Fathi, A., Laudenbach, F., Poenaru, V.: Travaux de Thurston sur les Surfaces. Asterisque, Vol. 66-67 (1979)

[FS] Flexor, M., Sentenac, P.: Preprint, Orsay

[G] Gilman, J.: Structure of elliptic irreducible subgroups of the modular group. Proc. Lond. Math. Soc. 47, 27-42 (1983) 
[Ker] Kerckhoff, S.: The Nielsen realization problem. Ann. Math. 117, 235-265 (1983)

[MSS] Mane, R., Sad, P., Sullivan, D.: On the dynamics of rational maps. Ann. Sci. Ec. Norm. Super. 16, 193-217 (1983)

[Mc1] McMullen, C.: Families of rational maps and iterative root-finding algorithms. Ann. Math. $125,467-493$ (1987)

[Mc2] McMullen, C.: Automorphisms of rational maps. (To appear, MSRI Geometric Function Theory Proceedings)

[SS] Shub, M., Smale, S.: On the existence of generally convergent algorithms. J. Complex. 2, 2-11 (1986)

[Sm] Smale, S.: On the efficiency of algorithms of analysis. Bull. AMS 13, 87-121 (1985)

[Sul1] Sullivan, D.: Conformal dynamical systems. In: Geometric Dynamics. (Lect. Notes Math., vol. 1007, pp. 725-752) Berlin Heidelberg New York: Springer 1983

[Sul2] Sullivan, D.: Quasiconformal homeomorphisms and dynamics III: Topological conjugacy classes of analytic endomorphisms (Preprint)

[ST] Sullivan, D., Thurston, W.: Extending holomorphic motions. Acta Math. 157, 243-258 (1986)

[W] Whyburn, G.: Analytic Topology. AMS Coll. Publ. 28, 1942

[Z] Zieschang, H.: Finite Groups of Mapping Classes of Surfaces. Lect. Notes Math. vol. 875. Berlin Heidelberg New York: Springer 1981 